\title{
Deep-water waves: On the nonlinear Schrödinger equation and its solutions
}

\author{
Nikolay K. Vitanov ${ }^{1 *}$, Amin Chabchoub ${ }^{2}$, Norbert Hoffmann ${ }^{2}$ \\ ${ }^{1}$ Institute of Mechanics, Bulgarian Academy of Sciences, \\ Acad. G. Bonchev, Bl. 4, 1113 Sofia, Bulgaria \\ 2 Institute of Mechanics and Ocean Engineering, Hamburg \\ University of Technology, 21073 Hamburg, Germany
}

\begin{abstract}
We present a brief discussion on the nonlinear Schrödinger equation for modeling the propagation of the deep-water wavetrains and a discussion on its doubly-localized breather solutions that can be connected to the sudden formation of extreme waves, also known as rogue waves or freak waves.
\end{abstract}

KEY WORDS: deep-water waves, Nonlinear Schrödinger equation, Dysthe equation, Akhmediev-Peregrine breathers, rogue waves

*corresponding author, e-mail: vitanov@imbm.bas.bg 


\section{Introduction}

Nonlinear phenomena are much studied in various area of science [1]-[6]. Many of these phenomena are modeled by nonlinear partial differential equations or systems of such equations, which increased the interest in the methods of obtaining exact and approximate solutions of such equations [7]-[11]. In this brief report we shall be interested in extreme deep-water phenomena, also referred to as freak or rogue waves [12, 13] and its description by breather solutions of the nonlinear Schrödinger equation. Rogue water waves are extreme high sea waves that can cause severe damage on commercial and other ships or on oil platforms. Such waves are deep-water waves which probably can be described by breather solutions of equations that belong to the family of the nonlinear Schrödinger equation. The latter will determine the scope of our report as follows: (I) short notices on linear and nonlinear surface gravity water water; (II) Heuristic derivation the nonlinear Schrödinger equation (NSE) for deep-water waves; (III) discussion on one extension of this equation (known as Dysthe equation); and (IV) a short discussion on breather solutions of the NSE obtained by Peregrine as well as Akhmediev and coworkers, which could be considered as appropriate models for the freak waves.

\section{Model equations for water waves: incom- pressible inviscid approximation}

We start from the differential form the basic equation of fluid mechanics are as follows [17]

$$
\begin{aligned}
\frac{\partial \rho}{\partial t}+\nabla \cdot(\rho \vec{v}) & =0: \text { mass conservation, } \\
\text { (1) } \frac{\partial \vec{v}}{\partial t}+(\vec{v} \cdot \nabla) \vec{v} & =-\frac{1}{\rho} \nabla p+\vec{f}+\frac{\eta^{*}}{\rho} \nabla^{2} \vec{v}: \text { momentum conservation. }
\end{aligned}
$$

In Eqs (1) $\rho$ is the density of the fluid; $\vec{v}$ is the fluid velocity; $p$ is the pressure; $\vec{f}$ summarizes the body forces acting on the fluid; $\eta^{*}$ is coefficient of viscosity called kinematic viscosity.

In addition boundary conditions should be imposed. We assume that the depth of the fluid is $h$ and it is bounded from below by a hard horizontal bed. The upper fluid surface is assumed to be free. The unperturbed free upper surface is at $z=0$. When the upper surface is perturbed, there is vertical displacement $\eta(x, y, t)$ of each point of the surface. Then, the 
boundary condition on the upper fluid surface is at $z=\eta(x, y, t)$. On the lower solid surface the normal component of velocity has to vanish, i.e. no flux is permitted at the bottom. That is, $v_{z}=0$ at $z=-h$.

We shall discuss the case of constant fluid density $\rho=$ const (incompressible fluid approximation). In addition, the waves will have wavelength much longer than approximately $1.8 \mathrm{~cm}$. For this case the viscosity effects are negligible, i.e., we shall consider gravity waves (and not capillary waves).

\subsection{Irrotational approximation and model of small am- plitude surface gravity waves}

The quantity $\vec{\omega}=\nabla \times \vec{v}$ is called vorticity of the flow and when $\vec{\omega}=0$ the flow is called irrotational. In this case the velocity of the flow is a potential field: $\vec{v}=\nabla \phi$, where $\phi$ is the velocity potential (the central quantity we shall discuss below). In addition, for gravity waves $\vec{f}=-g \vec{e}_{z}$, where $g$ is the acceleration of gravity and $\vec{e}_{z}=(0,0,1)$. For small water surface amplitudes and in the irrotational approximation, the model equations (1) and the boundary conditions are reduced as follows

$$
\begin{aligned}
\nabla^{2} \phi= & 0 ;-h<z<\eta(x, y, t) \rightarrow \text { from mass conservation, } \\
\frac{\partial \phi}{\partial t}=- & -\left[\frac{1}{2}\left(\left(\frac{\partial \phi}{\partial x}\right)^{2}+\left(\frac{\partial \phi}{\partial y}\right)^{2}+\left(\frac{\partial \phi}{\partial z}\right)^{2}\right)+\eta g\right] ; \text { at } z=\eta(x, y, t) \rightarrow \text { from } \\
& \text { momentum conservation, } \\
\frac{\partial \phi}{\partial z}= & \frac{\partial \eta}{\partial x} \frac{\partial \phi}{\partial x}+\frac{\partial \eta}{\partial y} \frac{\partial \phi}{\partial y}+\frac{\partial \eta}{\partial z} \frac{\partial \phi}{\partial z} ; \text { at } z=\eta(x, y, t) \rightarrow \text { from b.c. on } \\
& \text { the top surface, } \\
\frac{\partial \phi}{\partial z}= & 0 ; \text { at } z=-h \rightarrow \text { from b.c. on the bottom surface. }
\end{aligned}
$$

Thus, the model equation becomes linear (the Laplace equation) but the boundary conditions are nonlinear.

\subsection{Shallow-water waves and deep-water waves}

For small amplitudes (but long wavelengths) water waves, the nonlinear relationships from the system (2) can be linearized. If the mean surface displacement and the mean velocity potential are small with respect to the wavelength and to wave period scales, then, the nonlinear terms in the boundary conditions can be neglected. After a Taylor series expansion of the small 
quantity $\eta$ (and keeping only the first term of the expansion) the top boundary condition can be written as condition on $z=0$. Thus, we obtain the following simplified (and linear) problem

$$
\begin{aligned}
\nabla^{2} \phi & =0 ;-h<z<0, \\
\frac{\partial \phi}{\partial t} & =-g \frac{\partial \phi}{\partial z} ; \text { at } z=0 \\
\frac{\partial \phi}{\partial z} & =0 ; \text { at } z=-h .
\end{aligned}
$$

The next approximation is that we assume that the waves propagate in the $x$-direction and are uniform in the $y$-direction. Thus, the problem becomes one-dimensional and one searches for a traveling wave solution with wave frequency $\omega$ and wavenumber $k$ :

$$
\phi(x, t)=\bar{A}(x, z) \sin (k x-\omega t) .
$$

The substitution of Eq. (4) in (3) leads to the following solutions for the velocity potential $\phi$ and for the surface displacement $\eta$

$$
\begin{array}{r}
\eta=A \cos (k x-\omega t) ; \phi=\omega A \frac{\cosh (k(z+h))}{k \sinh (k h)} \sin (k x-\omega t) ; \\
A=2 \frac{a k}{\omega} \exp (-k h) \sinh (k h),
\end{array}
$$

where $a$ is a constant of integration, $A, k$ and $\omega$ denote the wave amplitude, the wavenumber and the wave frequency, respectively. The dispersion relation for the small amplitude surface water waves as well as their phase velocity $v$ and group velocity $v_{g}$ are as follows

(6) $\omega^{2}=g k \tanh (k h) ; v=\sqrt{\frac{g}{k} \tanh (k h)} ; v_{g}=\frac{v}{2}\left[1+\frac{2 k h}{2 \sinh (2 k h)}\right]$.

The relationship $R=\frac{\text { depth }}{\text { wavelength }}=\frac{h}{\lambda}$ has two limit cases: $R<<1$ (shallowwater waves) and $R>>1$ (deep-water waves). For the case of shallow-water waves the dispersion relation (6) can be approximated by

$$
\omega=k \sqrt{g h}\left[1-\frac{k^{2} h^{2}}{6}+\ldots\right] ; c_{0}=\sqrt{g h} .
$$

For very long shallow water waves $\omega=k c_{0} ; v=\frac{\omega}{k} \approx c_{0} ; v_{g}=\frac{\partial \omega}{\partial k} \approx k_{0}$. For deep-water waves the approximation for the dispersion relation is $\omega \approx \sqrt{g k}$ and the phase and group velocity are $v=\sqrt{g / k} ; v_{g}=\sqrt{g /(2 k)}$, respectively. That is, the group velocity is smaller and half the phase velocity. 


\section{Deep-water waves. The nonlinear Schrödinger equation. The Dysthe equation}

A weakly nonlinear model for shallow-water waves can be described by the Korteweg-de Vries equation. We shall be interested in the deep-water case, which can be modeled by the nonlinear Schrödinger equation.

\subsection{Derivation of the nonlinear Schrödinger equation by applying a Taylor series expansion to the dis- persion relation for deep-water waves}

A weakly nonlinear approximation to the nonlinear deep-water wave problem are the Stokes waves. They however are unstable against modulation perturbations. The velocity of the Stokes waves to second-order in steepness is

$$
v=\sqrt{\frac{g}{k}\left(1+\frac{k^{2} a^{2}}{2}\right)},
$$

where $a$ denotes now the wave amplitude. From Eq. (7) one obtains easily the dispersion relation $\omega=\sqrt{g k\left(1+\frac{k^{2} a^{2}}{2}\right)}$. Let us consider a slowly modulated Stokes wave wavetrain

$$
\eta=\operatorname{Re}\left[A(X, T) \exp \left(i\left(\omega_{0} t-k_{0} x\right)\right)\right]
$$

where $\omega_{0}$ and $k_{0}$ are the frequency and wave number of carrier Stokes wave and $A(X, T)$ is the modulation amplitude of the wavetrain. In addition, $X=\epsilon x$ and $T=\epsilon t(\epsilon<<1)$ are the slowly varying space and time variables, respectively. Physically, $\varepsilon:=A k_{0}$ is the steepness of the wave and is assumed to be small. Let us now perform a Taylor series expansion around the wavenumber $k_{0}$ and the amplitude $A_{0}=A(0,0) \ldots$ The dispersion relation of the carrier Stokes wave is

$$
\omega=\sqrt{g k\left(1+k^{2}|A|^{2}\right)},
$$

where $|A|$ is the amplitude of the Stokes wave (and the amplitude of the envelope). The Taylor series expansion about the wavenumber $k_{0}$ of the carrier wave and about the envelope amplitude $A=A_{0}=0$ as follows [17]

(10) $\omega=\omega_{0}+\frac{\partial \omega}{\partial k}\left(k-k_{0}\right)+\frac{1}{2} \frac{\partial^{2} \omega}{\partial k^{2}}\left(k-k_{0}\right)^{2}+\frac{\partial \omega}{\partial|A|^{2}}\left(|A|^{2}-\left|A_{0}\right|^{2}\right)$.

Let $\Omega=\omega-\omega_{0}$ and $K=k-k_{0}$. In addition (accounting also for Eq. (9p)) $\left.\frac{\partial \omega}{\partial k}\right|_{k=k_{0}}=v_{g}=\frac{\omega_{0}}{2 k_{0}}$ (note that the group velocity of the envelope is twice 
smaller than the phase velocity of the carrier wave); $\left.\frac{\partial^{2} \omega}{\partial k^{2}}\right|_{k=k_{0}}=2 P=-\frac{\omega_{0}}{8 k_{0}^{2}}$; $Q=\left.\frac{\partial \omega}{\partial|A|^{2}}\right|_{A_{0}=0}=\frac{1}{2} \omega_{0} k_{0}^{2}$. Then, from Eq. 10

$$
\Omega=v_{g} K+P K^{2}+Q|A|^{2} .
$$

The Fourier and the inverse Fourier transform of the envelope function are

$$
\begin{array}{r}
A(K, \Omega)=\mathcal{F}[A(X, T)]=\int_{-\infty}^{\infty} d X d T A(X, T) \exp [i(\Omega T-K X)] \\
A(X, T)=\mathcal{F}^{-1}[A(K, \Omega)]=\left(\frac{1}{2 \pi}\right)^{2} \int_{-\infty}^{\infty} d K d \Omega A(K, \Omega) \exp [-i(\Omega T-K X)] .
\end{array}
$$

From Eqs. 12

$$
\frac{\partial A}{\partial X}=i K \mathcal{F}^{-1}[A(K, \Omega)], \frac{\partial A}{\partial t}=-i \Omega \mathcal{F}^{-1}[A(K, \Omega)] .
$$

$\Omega$ and $K$ are of order $\epsilon$. Then from Eq.(13) we can write

$$
K=-i \epsilon \frac{\partial}{\partial X} ; \Omega=i \epsilon \frac{\partial}{\partial T} \text {. }
$$

The substitution of the relationships from Eq.(14) in Eq.(11) and application of the resulting operator equation to the envelope amplitude $A$ leads to the nonlinear Schrödinger equation for the evolution of the amplitude of the envelope of the wavetrain ( $\epsilon$ is incorporated in $T$ and $X$ by appropriate rescaling).

$$
i\left(\frac{\partial A}{\partial T}+\frac{\omega_{0}}{2 k_{0}} \frac{\partial A}{\partial X}\right)-\frac{\omega_{0}}{8 k_{0}^{2}} \frac{\partial^{2} A}{\partial X^{2}}-\frac{1}{2} \omega_{0} k_{0}^{2}|A|^{2} A=0 .
$$

Eq. 15 can be rescalled as follows: $\tau=-\frac{\omega_{0}}{8 k_{0}^{2}} T ; \xi=X-v_{g} T=X-\frac{\omega_{0}}{2 k_{0}} T$ (coordinate is in a frame that moves with the group velocity of the wavetrain); $q=\sqrt{2} k_{0}^{2} A$. The rescalled form of the nonlinear Schrödinger equation is

$$
i \frac{\partial q}{\partial \tau}+\frac{\partial^{2} q}{\partial \xi^{2}}+2|q|^{2} q=0
$$

\subsection{More accurate deep-water wave envelope equation: The Dysthe equation}

The Dysthe equation [18] is an extension of the nonlinear Schrödinger equation. This equation aims to solve the problem with the bandwidth limitation 
of the NSE. The nonlinear Schördinger equation is valid for small steepness values. i.e. $k_{0} A<<1$ and when the bandwidth is narrow $(\Delta k / k<<1$, $\Delta k$ is the modulation wavenumber). In order to obtain the Dysthe equation one starts with the model equations for the velocity potential $\phi(x, y, z, t)$ and surface displacement $\eta(x, y, t)$ for an incompressible inviscid fluid with uniform depth $h$

$$
\begin{aligned}
& \nabla^{2} \phi=0, \text { for }-h<z<\eta, \\
& \frac{\partial^{2} \phi}{\partial t^{2}}+g \frac{\partial \phi}{\partial z}+\frac{\partial}{\partial t}(\nabla \phi)^{2}+\frac{1}{2} \nabla \phi \cdot \nabla(\nabla \phi)^{2}=0, \text { at } z=\eta, \\
& \frac{\partial h}{\partial t}+\nabla \phi \cdot \nabla h=\frac{\partial \phi}{\partial z}, z=h, \\
& \frac{\partial \phi}{\partial z}=0, \text { at } z=-h \text {. }
\end{aligned}
$$

Next one assumes that $k A=O(\epsilon) ; \frac{\Delta k}{k}=O(\epsilon)^{1 / 2},(k h)^{-1}=O\left(\epsilon^{1 / 2}\right)$ and performs the following expansion for the velocity potential $\phi$ and surface displacement $h$

$$
\begin{aligned}
& \phi=\bar{\phi}+\frac{1}{2}\left(A \exp (i \theta+k z)+A_{2} \exp (2(i \theta+k z))+\ldots+\text { c.c. }\right), \\
& h=\bar{h}+\frac{1}{2}\left(B \exp (i \theta+k z)+B_{2} \exp (2(i \theta+k z))+\ldots+\text { c.c. }\right),
\end{aligned}
$$

where $\theta=k x-\omega t$. The drift $\bar{\phi}$, the set down $\bar{h}$ and the amplitudes $A, A_{2}, \ldots, B, B_{2}, \ldots$ are functions of the slow modulation variables $\epsilon x, \epsilon y, \epsilon t$. Furthermore, a non-dimensionalization of the variables is performed as follows: $\omega t \rightarrow t ; k(x, y, z) \rightarrow(x, y, z) ; k^{2} \omega^{-1}\left(A, \ldots, A_{n}, \bar{\phi}\right) \rightarrow\left(A, \ldots, A_{n}, \bar{\phi}\right)$; $k\left(B, \ldots, B_{n}, \bar{\eta}\right) \rightarrow\left(B, \ldots, B_{n}, \bar{\eta}\right)$. Note that the functions $\bar{\phi} ; \bar{\eta} ; A, \ldots, A_{n} ;$ $B, \ldots, B_{n}$ are functions of the variables $\epsilon^{1 / 2}(x, z, t)$. Proceeding with the expansions up to order $O\left(\epsilon^{3.5}\right)$, one obtains the Dysthe equation

$$
\begin{array}{r}
\frac{\partial A}{\partial t}+\frac{1}{2} \frac{\partial A}{\partial x}+\frac{i}{8} \frac{\partial^{2} A}{\partial x^{2}}-\frac{i}{4} \frac{\partial^{2} A}{\partial y^{2}}-\frac{1}{16} \frac{\partial^{2} A}{\partial x^{3}}+\frac{3}{8} \frac{\partial^{3} A}{\partial x \partial y^{2}}-\frac{5 i}{128} \frac{\partial^{4} A}{\partial x^{4}}+ \\
\frac{15 i}{32} \frac{\partial^{4} A}{\partial x^{2} \partial y^{2}}-\frac{3 i}{32} \frac{\partial^{4} A}{\partial y^{4}}+\frac{i}{2}|A|^{2} A+\frac{7}{256} \frac{\partial^{5} A}{\partial x^{5}}-\frac{35}{64} \frac{\partial^{5} A}{\partial x^{3} \partial y^{2}}+\frac{21}{64} \frac{\partial^{5} A}{\partial x \partial y^{4}}+ \\
\frac{3}{2}|A|^{2} \frac{\partial A}{\partial x}-\frac{1}{4} A^{2} \frac{\partial A^{*}}{\partial x}+i A \frac{\partial \bar{\phi}}{\partial x}=0
\end{array}
$$

for the corresponding boundary conditions

$$
\nabla^{2} \bar{\phi}=0, \text { for }-h<z<0,
$$




$$
\begin{array}{r}
\frac{\partial \bar{\phi}}{\partial z}=\frac{1}{2} \frac{\partial|A|^{2}}{\partial x}, \text { at } z=0 \\
\frac{\partial \bar{\phi}}{\partial z}=0, \text { at } z=-h .
\end{array}
$$

\section{Peregrine and Akhmediev-Peregrine breathers}

Akhmediev and co-workers [14, 15] derived a family of space-periodic pulsating solutions of the NSE, also called Akhemdiev breathers, that start from the plane wave solution at $\tau=-\infty$ and return again to the plane wave form at $\tau=\infty$. Taking this period to infinity, we obtain the Peregrine breather [16], a solution which is doubly-localized, in space and time, and pulsates only once. These properties make the Peregrine breather an ideal model to describe oceanic rogue waves. In the same work [14, Akhmediev and co-workers derived a higher-order doubly-localized breathers, also referred to the Akhmediev-Peregrine breather. In fact, there is an infinite hierarchy of doubly-localized Akhmediev-Peregrine solutions. Generally, the $j-$ th Akhmediev-Peregrine solution can be written in terms of polynomials:

$$
q_{j}(\xi, \tau)=q_{0} \exp \left(2 i\left|q_{0}\right|^{2} \tau\right)\left[(-1)^{j}+\frac{G_{j}+i H_{j}}{D_{j}}\right],
$$

where $q_{0}$ is proportional to the carrier wave amplitude and $G_{j}(\xi, \tau) ; H_{j}(\xi, \tau)$ and $D_{j}(\xi, \tau)$ are appropriate polynomials. The first-order rational solution (Peregrine breather) is given by

$$
G_{1}=4 ; H_{1}=16\left|q_{0}\right|^{2} \tau ; D_{1}=1+4\left|q_{0}\right|^{2} \xi^{2}+16\left|q_{0}\right|^{4} \tau^{2}
$$

The second-order rational solution (Akhmediev-Peregrine breather) is given by

$$
\begin{array}{r}
G_{2}=\left(|q|^{2} \xi^{2}+4\left|q_{0}\right|^{4} \tau^{2}+\frac{3}{4}\right)\left(\left|q_{0}\right|^{2} \xi^{2}+20\left|q_{0}\right|^{4} \tau^{4}+\frac{3}{4}\right)-\frac{3}{4} \\
H_{2}=2\left|q_{0}\right|^{2} \tau\left(4\left|q_{0}\right|^{4} \tau^{2}-3\left|q_{0}\right|^{2} \xi^{2}\right)+2\left|q_{0}\right|^{2} \tau\left(\left(2\left|q_{0}\right|^{2} \xi^{2}+\right.\right. \\
\left.\left.4\left|q_{0}\right|^{4} \tau^{2}\right)^{2}-\frac{15}{8}\right) \\
D_{2}=\frac{1}{3}\left(\left|q_{0}\right|^{2} \xi^{2}+4\left|q_{0}\right|^{4} \tau^{2}\right)^{3}+\frac{1}{4}\left(\left|q_{0}\right|^{2} \xi^{2}-12\left|q_{0}\right|^{4} \tau^{2}\right)^{2}+ \\
\frac{3}{64}\left(12\left|q_{0}\right|^{2} \xi^{2}+176\left|q_{0}\right|^{4} \tau^{2}+1\right)
\end{array}
$$

Figure 1 shows the two solutions. The Peregrine and Akhmediev-Peregrine 

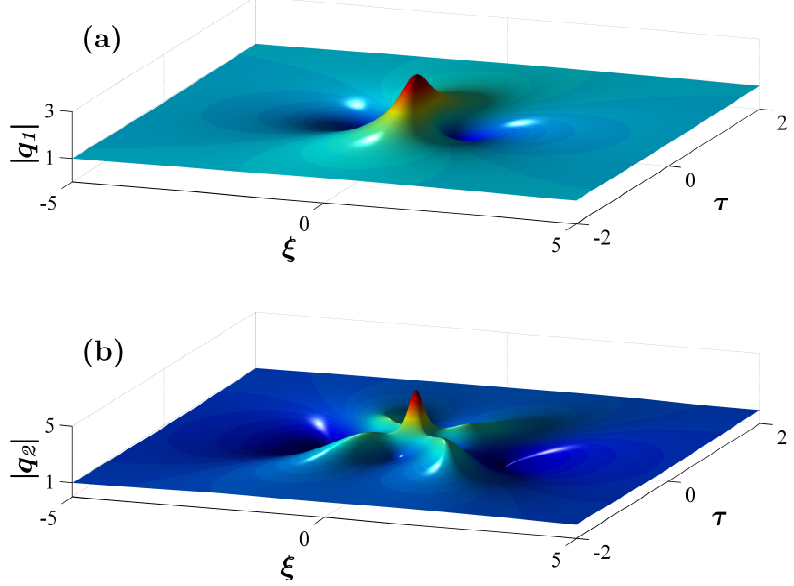

Figure 1: (a) First-order rational solution (Peregrine breather), which amplifies the carrier-amplitude by a factor of three. (b) Second-order rational solution (Akhmediev-Peregrine breather), which amplifies the carrier-amplitude by a factor of five. Both solutions are localized in both, space and time.

breathers up to fifth-order have been recently observed in a deep-water waves flume [19]-[22]. We intend to conduct in the future theoretical, numerical and experimental investigations of NSE solutions as well as other equations of this kind, based on the method of simplest equation [23]-[25] and similar.

\section{References}

[1] SCOTT, A. C. Nonlinear Science. Emergence and Dynamics of Coherent Structures. Oxford, UK, Oxford University Press, 1999.

[2] PANCHEV S., T. SPASSOVA, N.K. VITANOV. Analytical and numerical investigation of two families of Lorenz-like dynamical systems. Chaos, Solitons \& Fractals 33 (2007) 1658-1671.

[3] KANTZ H., D. HOLSTEIN, M. RAGWITZ, N. K. VITANOV. Markov Chain Model for Turbulent Wind Speed Data. Physica A 342 (2004) $315-321$.

[4] BOECK T., N. K. VITANOV. Low-Dimensional Chaos in Zero-PrandtlNumber Benard-Marangoni Convection. Phys. Rev. E 65 (2002) Article No:037203. 
[5] VITANOV N. K, I. P. JORDANOV, Z. I. DIMITROVA On Nonlinear Population Waves, Applied Mathematics and Computation 215 (2009) 2950-2964.

[6] VITANOV N. K. Upper Bounds on the Heat Transport in a Porous Layer. Physica D 136 (2000) 322 - 339.

[7] AKHMEDIEV N. N., A. ANKIEWICZ. Solitons. Nonlinear Pulses and Beams. London, Chapman \& Hall, 1997.

[8] HIROTA, R. Exact Solution of Korteweg-de Vries Equation for Multiple Collisions of Solitons. Phys. Rev. Lett., 27 (1971) 1192 - 1194.

[9] KUDRYASHOV, N. A., M. B. SOUKHAREV. Popular Ansatz Methods and Solitary Wave Solutions of the Kuramoto - Sivashinsky Equation. Regular \& Chaotic Dynamics 14 (2009) 407 - 419.

[10] VITANOV N. K. Modified Method of Simplest Equation: Powerful Tool for Obtaining Exact and Approximate Traveling-Wave Solutions of Nonlinear PDEs. Commun. Nonlinear Sci. Numer. Simulat. 16 (2011) 1176 $-1185$.

[11] VITANOV, N. K., Z. I. DIMITROVA, H. KANTZ Modified method of simplest equation and its application to nonlinear PDEs. Applied Mathematics and Computation, 216 (2010) 2587-2595.

[12] KHARIF C., E. PELINOVSKY, A. SLUNYAEV. Rogue waves in the ocean. Berlin, Sprinfer, 2009.

[13] PELINOVSKY E., C. KHARIF. (Eds.) Extreme ocean waves. Berlin, Springer, 2008.

[14] AKHMEDIEV N., V. M. ELEONSKII, N. E. KULAGIN. Generation of of a periodic sequence of picosecond pulses in an optical fiber. Exact solutions. Sov. Phys. JETP 89 (1985) 1542-1551.

[15] AKHMEDIEV N., V. I. KORNEEV. Modulation instability and periodic solutions of the nonlinear Schrödinger equation. Theor. Math. Phys. (USSR) 69 (1987) 1089-1093.

[16] PEREGRINE, D. H. Water waves, nonlinear Schrdinger equations and their solutions. J. Austral. Math. Soc. B 25 (1983) 16-43.

[17] REMOISSEnET M. Waves Called Solitons. Berlin, Springer, 1993. 
[18] DYSTHE K. B. Note on a modification to the nonlinear Schrödinger equation for application to deep water waves. Proc. Roy. Soc. London, Ser. A 369 (1979), 105-114.

[19] CHABCHOUB A., N. VITANOV, N. HOFFMANN. Experimental evidence for breather type dynamics in freak waves. PAMM 10, No. 1 (2010) 495-496.

[20] CHABCHOUB A., N. P. NOFFMANN, N. AKHMEDIEV N. Rogue wave observation in a water wave tank. Physical Review Letters 106, No. 20, (2011), Article No. 204502

[21] CHABChOUB A., N. P. NOFFMAnN, M. OnORATO, N. AKHMEDIEV. Super Rogue Waves: Observation of a Higher-Order Breather in Water Waves. Physical Review X 2 No.1 (2012), Article No. 011015.

[22] CHABChOuB A., N. HOFFMAnN, M. OnORATO, A. SLUNYAEV, A. SERGEEVA, E. PELINOVSKY, N. AKHMEDIEV. Observation of a hierarchy of up to fifth-order rogue waves in a water tank. Physical Review E 86 No.1 (2012), Article No. 056601.

[23] VITANOV N. K., Z. I. DIMITROVA. Application of the Method of Simplest Equation for Obtaining Exact Traveling-Wave Solutions for Two Classes of Model PDEs From Ecology and Population Dynamics. Commun. Nonlinear Sci. Numer. Simulat. 15 (2010) 2836-2845.

[24] VITANOV N. K. Application of Simplest Equations of Bernoulli and Riccati kind for Obtaining Exact Traveling Wave Solutions for a Class of PDEs with Polynomial Nonlinearity. Commun. Nonlinear Sci. Numer. Simulat. 15 (2010) 2050 - 2060.

[25] VItANOV N. K., Z. I. DIMITROVA, N. K. VITANOV. On The Class of Nonlinear PDEs that can be Treated by the Modified Method of Simplest Equation. Application to Generalized Degasperis-Processi Equation and b-Equation. Commun. Nonlinear Sci. Numer. Simulat. 16 (2011) 3033 3044 . 